\title{
Research on My Country's Private Investment Scale Efficiency Based on Solow Model
}

\author{
Sun $\operatorname{Min}^{1, \text { a }}$ \\ ${ }^{1}$ School of Economics and Management of XI'an Mingde Institute of Technology, Xi'an, Shaanxi, China \\ a33744205@qq.com
}

\begin{abstract}
In my country's economic development, government investment has always been an important political and economic means of stimulating economic growth. Government investment is also the fastest and most effective way to promote economic development. For example, after the subprime mortgage crisis in the United States, my country resumed stimulating economic growth in 2008. The 4 trillion government investment invested in the market has played an important role in the recovery and development of my country's economy. However, we should see that while government investment brings many benefits to economic development, it also has limitations. For example, government investment involves too many areas; the investment structure is relatively simple. Therefore, relying solely on government investment cannot fully achieve the expected Economic goals.

In the past 40 years of reform and opening up, private investment in the form of private enterprises has been greatly developed, and private investment has gradually replaced state-owned investment as the main force in my country's fixed asset investment. Although my country's private investment has achieved tremendous growth in quantity, there are still some problems in its investment efficiency. At present, the investment efficiency of my country's private investment is still at a relatively low level, and the improvement of the efficiency of private investment is crucial to the development of my country's economy. Therefore, this article studies the efficiency of my country's private investment from the perspective of scale efficiency, and concludes that my country's private investment is inefficient due to insufficient scale investment. In the future, the scale of private investment should be further increased to achieve scale efficiency.
\end{abstract}

Keywords: Private investment, Scale, Efficiency

\section{基于索洛模型的我国民间投资规模效率研究}

\author{
孙敏 ${ }^{1, a}$ \\ ${ }^{1}$ 西安明德理工学院经济与管理学院, 西安, 陕西, 中国 \\ a33744205@qq.com
}

\section{摘要}

在我国的经济发展中, 政府投资一直是拉动经济增长的重要政治经济手段, 政府投资也是促进经济发展最快 速最有效的方式，如美国次贷危机之后，我国为恢复刺激经济增长，于 2008 年向市场投放了 4 万亿的政府投 资, 对我国经济的恢复发展起到了不可忽视的作用。但是我们应当看到政府投资在给经济发展带来诸多好处 的同时, 也存在局限性, 比如政府投资的涉及面太广; 投资结构较为单一等, 因此, 单纯依靠政府投资并不 能完全实现预期的经济目标。

改革开放四十年以来，以民营企业为主要形式的民间投资已经得到了极大的发展，民间投资已经逐步取代国 有投资成为我国固定资产投资的主力。虽然我国民间投资已经取得了数量上的巨大发展，但是其投资效率仍 然存在一些问题。目前我国民间投资的投资效率依然处于较低的水平，民间投资效率的提高对我国经济的发 
展至关重要。因此, 本文从规模效率角度研究我国民间投资效率问题, 得出我国民间投资存在规模投资不足 的非效率，在未来应进一步加大民间投资规模，实现规模效率。

关键词：民间投资；规模；效率

\section{1. 民间投资的界定}

目前学术界对民间资本的定义还没有统一的界 定，但从投资主体来看，对于民间投资的概念主要有 三种观点: 广义论认为除国有资本投资以外的投资都 是民间投资; 中性论认为民间投资是除国有、港澳台 和外商资本投资以外的投资; 狭义论则从相对公有制 的角度, 将民间投资定义为非公有制的投资, 即不包 括股份制企业、联营经济、集体经济、外资四大经济 成分, 专指个体经济与私营经济投资。本文采用目前 研究应用较多的中性论定义, 即民间投资是指一定区 域范围内的全社会资本投资中剔除国有、港澳台和外 商资本投资的投资。具体包括集体、个体、私营、联 营、股份制及其他经济类型的固定资产投资。其计算 方法可以从两个角度进行: 一是用全社会资产投资减 去国有投资、港澳台投资和外商投资部分; 二是直接 将集体、个体、私营、联营、股份制及其他经济类型 的资产投资相加而得。两种计算方法得到的民间投资 数额是一致的。

\section{2. 民间投资效率评价的思路}

学者们对投资效率测度方法的研究从根本上说 都是从投入与产出的对比关系角度展开的, 净现金流 准则法研究的是从总资本收益与总投资的对比; 边际 资本产出比研究的是增量资本 $(\triangle K)$ 与增量生产总值 $(\triangle \mathrm{Y})$ 的关系; 全要素生产率法是把投入要素具体分 为了资本、劳动和技术进行研究; 数据包络分析法属 于非参数前沿效率分析方法, 它是对多投入多产出决 策单元进行相对效率评价的计量经济方法; 资本配置 效率法是对行业结构投入与产出角度研究资本配置 效率的。在这五种方法中, 既有从投资总量角度研究 投资是否存在投资过度或投资不足问题，进而分析资 本投资的效率, 也有从投资的结构角度分析资本在不 同的行业及不同的地区分配是否合理进而分析投资 的效率; 既有从投资结果角度即投资的经济指标研究 投资效率, 也有从投资过程中是否采用了先进的技术 或经验来分析投资效率的。综合已有投资效率的研究 以及我国民间投资的实际发展特点, 本文利用生产函 数模型从投资总额角度研究民间投资的规模效率。

\section{3. 民间投资规模效率评价的理论基础}

在内生经济增长理论当中, 美国经济学家巴罗在 规模报酬不变的柯布-道格拉斯生产函数中引入了公
共部门变量, 从而建立出一个以公共部门支出为中心 的内生增长模型, 并得出了最优财政支出规模的计算 方法, 据此我们也可以借鉴此模型来探讨民间投资的 规模效率问题。根据巴罗的理论，国民经济生产函数 可以表示为:

$$
Y_{t}=\left(A L_{t}\right)^{\alpha} K_{t}^{\beta} G_{t}^{\gamma}
$$

其中 Yt 表示 GDP, A 反映技术水平 (全要素生产 率), Kt 表示民间投资, Lt 表示劳动力投入, Gt 表示 政府投资。式 (1) 两边同时对时间求导数, 并且同时 除以 Yt 可得:

$$
\begin{gathered}
\frac{Y_{t}}{Y_{t}}=\frac{\partial Y_{t}}{\partial L_{t}} * \frac{L_{t}^{\prime}}{Y_{t}}+\frac{\partial Y_{t}}{\partial K_{t}} * \frac{K_{t}^{\prime}}{Y_{t}}+\frac{\partial Y_{t}}{\partial G_{t}} * \frac{G_{t}^{\prime}}{Y_{t}} \\
=\frac{\partial Y_{t}}{\partial L_{t}} * \frac{L_{t}}{Y_{t}} * \frac{L_{t}^{\prime}}{L_{t}}+\frac{\partial Y_{t}}{\partial K_{t}} * \frac{K_{t}^{\prime}}{Y_{t}}+\frac{\partial Y_{t}}{\partial G_{t}} * \frac{G_{t}^{\prime}}{Y_{t}} \\
\text { 设 } \boldsymbol{a}=\frac{\partial \boldsymbol{Y}_{t}}{\partial \boldsymbol{L}_{t}} * \frac{\boldsymbol{L}_{t}}{\boldsymbol{Y}_{t}} \text { 为就业产出弹性, } M P K=\frac{\partial Y_{t}}{\partial K_{t}}
\end{gathered}
$$

为民间投资的边际产出弹性, $M P G=\frac{\partial Y_{t}}{\partial G_{t}}$ 为政府投 资的边际产出弹性, 根据边际收益等于边际成本等于 1 , 当 $\mathrm{MPK}=1$ 时, 民间投资达到最优规模, 因此, 设 $m=\frac{\partial Y_{t}}{\partial K_{t}} * \frac{K_{t}}{Y_{t}}$ 为民间投资产出弹性, $n=\frac{K_{t}}{Y_{t}}$ 为衡量民 间投资规模的性对指标，则 $M P K=\frac{m}{n}$ ，即

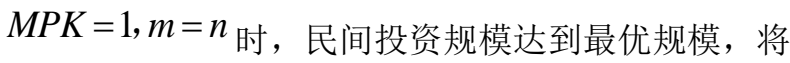
$\mathrm{m}$ 代入（2）可得,

$$
\frac{Y_{t}^{\prime}}{Y_{t}}=a \frac{L_{t}^{\prime}}{L_{t}}+m \frac{K_{t}^{\prime}}{Y_{t}}+M P G \frac{G_{t}^{\prime}}{Y_{t}}
$$

于是, $m$ 值的估计就是民间投资占国内生产总值 比例所表示的民间投资最优规模。

\section{4. 我国民间投资规模效率的现实状况}

改革开放以来, 我国民间资本呈现出了较快的增 长速度，无论是我国民间投资的总额还是相对额（民 间投资占全社会资产投资的比重)，其数量都是增加 的。从图 1 可以看到，我国民间投资总额在 1980 年 时只有 165 亿元, 占全社会资产投资的比例为 $18.1 \%$, 而在 2019 年时，民间投资总额达到 311159 亿元，与 1980 年相比增长达 1884 倍之多，民间投资占全社会 资产投资的比例达到 $55.48 \%$ ，比 1980 年足足增长 37.38 个百分点。图 1 还清晰的显示出我国民间投资 
占全社会资产投资的比重除受个别特殊年份的影响 外，基本上处于上升通道之中。其中，1993 年我国民 间投资占全社会资产投资的比重出现一个凸点，这主 要是由我国当年的房地产投资热潮引起的。至 2003 年我国民间投资额已经占据了全社会总投资额的大 半部分 (52.2\%)，之后其比例仍不断增加。我国民间 投资额占全社会投资额的比重越来越多意味着我国 的政府投资额在日益减少，这充分说明我国的民间投 资市场正向更加规范的方向发展, 在不断激烈的竞争 环境下表现出了更强的适应性, 对我国经济的增长发 挥着越来越重要的作用。图 2 可以清晰的反应出我国 民间投资增长率与宏观经济发展的关系，民间投资的 增长趋势与宏观经济的发展方向基本保持一致。

从民间投资发展的规模可以看到, 民间投资的发 展规模已经取得了较为突出的成就, 发展较为迅速, 但同时我们应当看到, 民间投资的规模壮大并不意味 着效率的提高, 我国长期存在的制度性障碍、法律法 规的缺失等一系列问题导致我国民间投资的发展潜 力并未真实体现出来，现实发展中的民间投资规模效 率的表现并不理想。

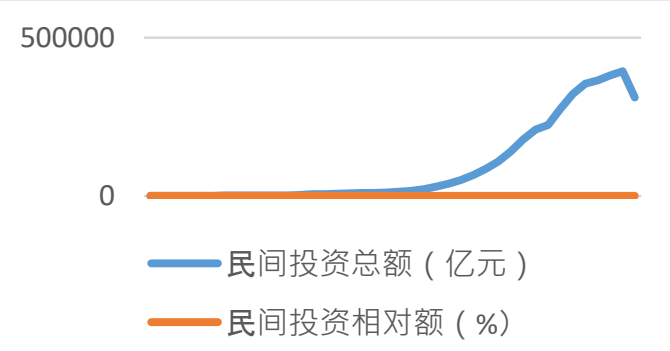

图 1 我国民间投资的总量与相对量

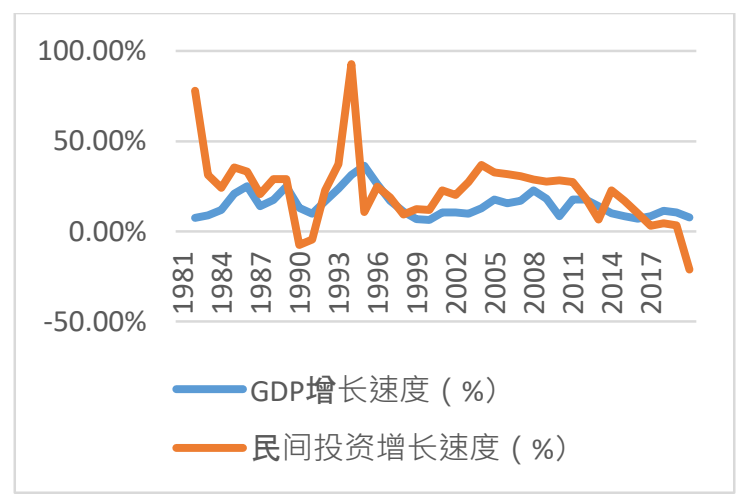

图 2 我国民间投资与 GDP 增长速度

\section{5. 民间投资规模效率的实证分析}

\section{1 基本模型的构建}

索洛模型所描述的投入产出关系用函数可以表 示为 $Y(t)=F[K(t), A(t) L(t)]$, (其中 $Y$ 表示产出, $\mathrm{K} 、 \mathrm{~L}$ 分别表示资本和劳动投入, $\mathrm{A}$ 表示知识或是劳动 有效性）。本文也采用生产函数的方法研究民间投资 的规模效率问题, 基本思路就是把索洛模型中的资本 分解为政府投资与民间投资，具体的函数形式为:

$$
Y_{t}=\left(A L_{t}\right)^{\alpha} K_{t}^{\beta} G_{t}^{\gamma}
$$

对 (4) 两边取对数可得,

$$
\operatorname{lnYt}=1 \mathrm{nA}+\mathrm{a} 1 \mathrm{nLt}+\beta 1 \mathrm{nKt}+\gamma \operatorname{lnGt}
$$

其中 $\beta=(\sigma \mathrm{Yt} / \sigma \mathrm{Kt})$

$K t / Y t=M P K(K t / Y t)$, 因此, 当 $M P K=1$ 时, $\beta$ 就是 用相对指标来表示的民间投资的最优规模。

\section{2 样本数据说明}

采用 1996-2019 年国家统计局网站公布的数据 对我国民间投资规模效率进行分析。其中，民间投资 数据仍是全社会固定资产投资中剔除国有、港澳台和 外资的部分，政府投资为全部投资数额减去民间投资 的剩余部分, 劳动力数据为每年的就业人口数量, 民 间投资、政府投资以及 GDP 的单位是亿元，劳动力的 单位是万人。

\section{3 单位根检验}

由于分析数据属于时间序列数据, 而在实际经济 运行当中, 时间序列数据大多是非平稳的, 使用非平 稳的数据极有可能出现 “伪回归” 问题, 因此, 在实 证分析之前需要检验数据的平稳性。本文采用 $\mathrm{ADF}$ 单 位根检验方法对各变量进行平稳性检验, 结果如表 1 所示:

\begin{tabular}{|c|c|c|c|c|}
\hline 序列 & 检验形式 & ADF 值 & $\mathrm{T}$ 统计值（1\%置信度） & 结论 \\
\hline $\operatorname{lnYt}$ & $(\mathrm{C}, 0,2)$ & -0.928836 & -3.699871 & 不平稳 \\
\hline $\operatorname{lnKt}$ & $(\mathrm{C}, 0,1)$ & -0.779000 & -3.661661 & 不平稳 \\
\hline $\operatorname{lnGt}$ & $(\mathrm{C}, 0,1)$ & -1.640449 & -3.670170 & 不平稳 \\
\hline $\operatorname{lnLt}$ & $(\mathrm{C}, 0,1)$ & -2.986416 & -3.661661 & 不平稳 \\
\hline
\end{tabular}

表 1 各变量的平稳性检验结果 


\begin{tabular}{lllll}
\hline DlnYt & $(\mathrm{C}, 0,2)$ & -4.662698 & -3.679322 & 平稳 \\
DlnKt & $(\mathrm{C}, 0,1)$ & -4.733961 & -3.670170 & 平稳 \\
DlnGt & $(\mathrm{C}, 0,1)$ & -3.822217 & -3.670170 & 平稳 \\
DlnLt & $(\mathrm{C}, 0,1)$ & -4.734030 & -3.670170 & 平稳 \\
\hline
\end{tabular}

说明: (C, 0, P) 表示回归方程中只包括常数项, 滞后阶数 P 根据 SIC 和 SC 准则确定。

检验结果表明, 原始序列中各变量对数值的 $\mathrm{ADF}$ 统计量都不小于 $1 \%$ 置信度下的临界值, 变量存在单 位根, 在时间序列上都是非平稳的。在对变量的对数 值进行一阶差分后, D lnYt、DlnKt、D lnLt、DlnGt 的 $\mathrm{ADF}$ 统计量都分别小于 $1 \%$ 置信度下的临界值, 一阶差 分序列是平稳的, 所以, 各变量都是 I (1) 过程, 满足 同阶单整的条件，可以继续进行协整检验。

\section{4 协整检验}

本文采用 Johansen 检验法进行协整检验。首先， 由 AR roots 均落在单位圆内我们得知 VAR 系统是稳 定的, 然后, 根据无约束 VAR 模型滞后阶数的选择标 准, 我们确定协整模型的滞后阶数是 2 期, 并且协整 检验形式为 “序列有线形趋势且协整方程仅含截距
项”。经检验, （见表 2）。由表 2 可知, 变量 $1 n Y t 、$ lnKt、 $1 n G t 、 1 n L t$ 之间存在协整关系, 并且在 5\%置 信水平下存在 2 个协整方程, 经过标准化之后, 得到 变量之间长期的均衡关系是:

$$
\begin{aligned}
\operatorname{lnYt}= & -7.194676+0.5118 \operatorname{lnKt}+0.2974 \operatorname{lnGt} \\
& +1.0506661 \mathrm{nLt}
\end{aligned}
$$

$1 n K t$ 的系数 0.5118 即是民间投资的产出弹性系 数, 即 $\mathrm{MPG}=1$ 时,民间投资占 GDP 的比重为 $51.18 \%$, 该比例就是民间投资的最优规模。根据协整方程我们 还可以看到，政府投资的产出弹性系数是 $29.74 \%$ ，民 间投资的产出弹性系数大于政府投资的产出弹性系 数，即民间投资的产出效率是高于政府投资的。

表 2 变量的协整检验结果

\begin{tabular}{llllll}
\hline 变量 & $\begin{array}{l}\text { 协整向量个 } \\
\text { 数 }\end{array}$ & $\begin{array}{l}\text { 迹统 } \\
\text { 计量 }\end{array}$ & 5\%临界值 & 最大特征值统计量 & 5\%临界值 \\
\hline $\begin{array}{l}\text { lnYt } \\
\operatorname{lnKt}\end{array}$ & 0 个* & 65.4172 & 47.85613 & 30.10617 & 27.58434 \\
$\operatorname{lnGt}$ & & & & & \\
$\operatorname{lnLt}$ & & & & &
\end{tabular}
最多 1 个*
35. 31102
29. 79707
20. 89925
14. 26460

注: *表示在 $5 \%$ 显著水平上拒绝原假设。

\section{6. 结论}

通过对变量 $1 n Y t 、 1 n K t 、 1 n G t 、 1 n L t$ 进行协整 检验, 我们验证了经济增长与民间投资之间存在着长 期的均衡关系, 并且得出民间投资的最优规模是民间 投资占 GDP 的 51. 18\%。图 3 显示了 1980 年到 2019 年 我国实际民间投资占 GDP 的比重, 从图中可以发现, 1980 年以来我国民间投资占 GDP 的比重基本保持上 升的态势，到 2018 年, 我国民间投资规模达到最大值, 民间投资规模占 GDP 的比重为 $42.87 \%$ 。虽然民间投 资规模在不断扩大, 但其还是低于最优规模 51. 18\%的, 这说明我国目前的民间投资仍然存在规模投资不足 的非效率。因此, 在未来的发展中应进一步挖掘民间 资本, 加大民间投资规模, 并采取有力的政策予以指 导，实现规模效率。

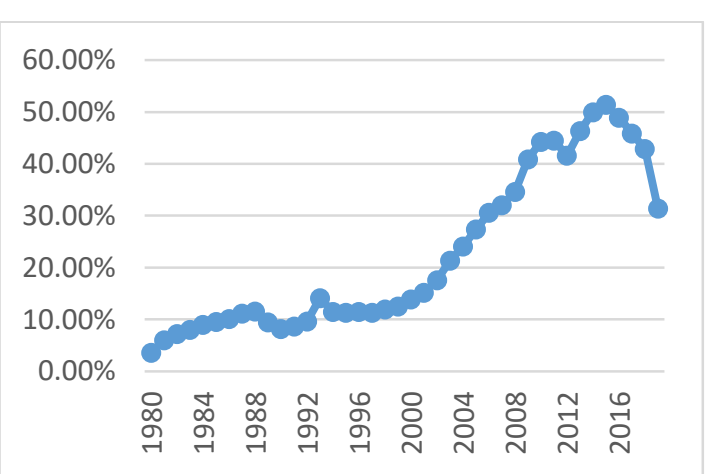

图3 1980-2019年我国民间投资占GDP的比重

\section{REFERENCES}

[1] Yu Jinkai, Shan Chunhong, An Empirical Analysis of the Efficiency of State-owned Investment and Private Investment in my country. Journal of Hebei University of Economics and Business, vol25, pp. 21-25, 2004 
[2] Robert J. Barro, Javier Saray Martin (2000). Economic Growth, China Social Sciences Press, Bei Jing

[3] Gao Hongye(2004), Western Economics(Second Edition), China Renmin University Press, Bei Jing

[4] Wu Chunye, Zhang Qiancheng, Return on Capital, Private Investment and Economic Growth-A Case Study of Wanjiang Region, Journal of Tongling University, 2020

[5] Han Yu,Hua Xiaoan, Research on the Correlation between Capital Allocation Efficiency and Financial Market-An Empirical Research Based on Shandong Province Data, Shandong Social Sciences, pp. 153-156, 2009 